\title{
Post-transplant pulmonary Coccidioidomycosis in a non-endemic area: Early diagnosis, the cornerstone for treatment of a life-threatening condition
}

\author{
Dalia Leyva-Córdova ${ }^{1}$, Omar Gabrie ${ }^{1}$, Torres-Valencia ${ }^{1}$, Guillermo Cárdenas-Membrila ${ }^{3}$, Paulino R Leal Villalpando ${ }^{1,2}$, Luis M Argote- \\ Green $^{4}$, Arturo Galindo-Fraga ${ }^{2}$ and Juan José Plata-Muñoz ${ }^{* *}$ \\ ${ }^{1}$ Escuela Nacional de Medicina y Ciencias de la Salud del Tecnológico de Monterrey, Campus Ciudad de México, México \\ ${ }^{2}$ Instituto Nacional de Ciencias Médicas y Nutrición "Salvador Zubirán”, Ciudad de México, México \\ ${ }^{3}$ Hospital General "Manuel Gea Gonzalez”, Ciudad de México, México \\ ${ }^{4}$ Clínica de Trasplante, Hospital Ángeles Acoxpa, Ciudad de México, México
}

\begin{abstract}
Coccidioidomycosis is an endemic mycosis in the southwest of the United States of America, northwestern Mexico and parts of Central and South America. Although this mycosis is infrequent outside the endemic zones and usually mild in immune competent individuals, it could be a lethal complication in the immunosuppressed patient. People who live in or travel to an endemic region can become infected and the time-frame for clinical appearance and severity correlates with the underlying immune status. In endemic regions, in which the levels of suspicion are high, this infection has been described after lung, kidney, heart and liver transplantation with an incidence of $1-7 \%$ and a concerning mortality rate of 20-50\%. In non-endemic areas, the low level of suspicion is associated with a delay in the diagnosis and an even higher morbi-mortality. This work presents a case of early post-transplant pulmonary coccidioidomycosis in a renal recipient who was born and lives in a non-endemic zone, in which an aggressive diagnostic approach allowed an early diagnosis and biopsy-driven anti-mycotic treatment. This case highlights the changing landscape of traditionally well-delimited endemic areas and the need for a higher level of suspicion when transplant patients develop non-specific sings of a postoperative pulmonary infection.
\end{abstract}

\section{Introduction}

Coccidioidomycosis is traditionally considered an endemic mycosis in the southwest of the United States of North America (US), selected areas of the northwest of Mexico, Central and South America [1,2]. This mycosis is originated by dimorphic, soil-dwelling, fungi which grow as saprobes in arid, alkaline soil of semi-desert regions. The Coocidioides ssp is responsible for Coccidioidomycosis or San Joaquin Valley Fever, an infectious disease characterized by a very broad spectrum of non-specific clinical manifestations [3,4]. Approximately $60 \%$ of the patients firstly exposed to Coccidioides ssp develop asymptomatic infections. In the remaining $40 \%$ the primary infection results in symptoms that range from a self-limited influenza-like illness to a relatively rare life-threatening disseminated disease which can require expensive and aggressive treatments, hospitalization and long-term monitoring [1,5-7]. An intact immune system is essential to control coccidioidomycosis infection; therefore, patients who have impaired immunity, especially cellular immunity, such as that caused by the human immunodeficiency virus or iatrogenic immunosuppression secondary to organ transplantation, may contract an infection with severe or life-threatening manifestations [8].

The traditional paradigm of coccidiodomycosis stresses that this mycosis is infrequent outside the endemic zones and explains why this disease could be a potentially lethal complication when the presence of this disease is not suspected, or the diagnosis and treatment are delayed in a patient living in a non-endemic zone, especially in an immunosuppressed patient. The aim of this work is to present a case of early post-transplant pulmonary coccidioidomycosis occurring in a recipient of a renal transplant who was born and lives in a nonendemic zone, in which an aggressive diagnostic protocol allowed an early diagnosis and a specific anti-mycotic treatment.

\section{Case presentation}

We report a case of a 58-year-old male with end-stage renal disease secondary to diabetes mellitus type 2 , treated with home-base peritoneal dialysis. The patient was born and lived in an urban area of the State of Mexico, a province situated 80 minutes to the north of Mexico City, right in the center of the country. The patient had a satisfactory metabolic control and adequate hygienic and nutritional habits. As a worker of a medium-size gas company he is in close contact with methane and metals and travels locally very often. He was a member of a hunting club, usually practicing the sport in the northern regions of Mexico, near the border with the United States, but he is not very active since his diagnosis of diabetes 15 years ago. He received a living-unrelated renal transplant from a healthy 36-year old female on November 2012. Conventional donor and recipient medical, surgical

*Correspondence to: Juan José Plata Muñoz, Clínica de Trasplante, Hospital Ángeles Acoxpa, Calzada Acoxpa 430, Colonia Ex-Hacienda Coapa, C.P. 14308 Delegación Tlalpan, Ciudad de México, México, Tel: +52 (55) 5677 9011; +52 (55) 5677 9122; +52 1 (55) 8564 5429; E-mail: jj.platamunoz@gmail.com

Received: November 26, 2018; Accepted: December 03, 2018; Published: December 07, 2018 

condition

and infectious pre-transplant evaluation protocols were followed, and the surgical procedure was authorized by the hospital's renal transplant committee.

The laparoscopic donor nephrectomy and the renal transplant were performed without complications and patients discharged 3 and 6 days after the surgical procedure, respectively. The kidney allograft was implanted using an end-to-side vascular anastomosis to the external iliac vessels and a double J catheter was used to stent the endto-side anastomosis between the ureter and the urinary bladder. The transplanted kidney had immediate graft function and post-transplant evolution was uneventful. The immunosuppression regime was initiated with pre-transplant administration of $20 \mathrm{mg}$ of basiliximab and continued with conventional triple immunosuppression therapy (1gm of tacrolimus, $600 \mathrm{mg}$ of mycophenolate mofetil, and $50 \mathrm{mg}$ of prednisone), an additional dose $20 \mathrm{mg}$ of basiliximab and prophylactic administration of trimethoprim/sulphamethoxazole and acyclovir was given post-transplant.

Three weeks after hospital discharge, the transplanted patient returned to the hospital because the appearance of malaise, generalized myalgia and diaphoresis, followed by dysuria, abdominal cramps, tenesmus and rectorrhagia. Muscle weakness and distal tremor of lower extremities, dehydration, diaphoresis, hypothermia, hypotension, an impetiginized ulcer in the labial commissure, as well as an abnormal discharge around the exit-site of the peritoneal dialysis catheter were documented but there was no sign of infection in the surgical site. Mild anemia and neutrophilia without leukocytosis, moderate elevation of $\mathrm{C}$ reactive protein and procalcitonin, normal levels of nitrogen-containing compounds, hyponatremia, hypomagnesemia and metabolic alkalosis were reported in his initial blood-tests. The postero-anterior chest X-rays showed non-specific images compatible with moderate pulmonary venous-capillary hypertension. Blood, urine, peritoneal fluid, exit-site of the peritoneal catheter and labial ulcer cultures were performed and empirical administration of meropenem, vancomycin and caspofungin was initiated. With the clinical suspicion of complicated urosepsis vs peritoneal catheter-associated peritonitis, ureteral and peritoneal catheters were removed immediately. Blood levels of tacrolimus were on the expected therapeutic range and the indicated dose was reduced by half while prednisone and mycophenolate mophetil was suspended.

After 48 hours of evolution, malaise, distal myalgia and episodes of diaphoresis, hypothermia and volume-responding hypotension continued. Urine test and peritoneal fluid were normal and the diagnosis of urosepsis and peritonitis were ruled-out. Levels of serum creatinine and nitrogen-containing compounds were normal but mild leukocytosis, elevated levels of CRP and pro-calcitonin persisted. Positive cultures from the exit-site of the catheter and blood confirmed the presence of Staphyloccocus Aureus methicillin-sensible and moxifloxacin was added to the treatment. Although an initial improvement in the general conditions of the patient was documented, the evening episodes of diaphoresis, hypothermia and hypotension persisted. A thoraco-abdominal CT scan showed a diffuse punctiform infiltrate in both lungs, highly suggestive of and opportunistic pulmonary infection and administration of liposomal amphotericin B to cover a possible pulmonary mycotic infection was initiated. After 72 hours of evolution, a video-assisted pulmonary biopsy was performed. Thoracoscopy showed a diffuse micro nodular lung infiltration and a wedge lung biopsy with a linear stapler was performed. There was no need of a chest tube, the patient was extubated without complications and its postoperative evolution was satisfactory. The lung biopsy confirmed the presence of pulmonary coccidioidomycosis.
Guided by the result of the pulmonary biopsy, specific therapy with IV fluconazole was initiated only after 4 days of initial hospitalization and the clinical condition of the patient gradually improved. Episodes of diaphoresis, hypothermia and hypotension completely disappeared after 48 hours of IV fluconazole administration and the patient was discharged after 11 days of hospitalization with no evidence of pulmonary complications, hemodynamically stable and with normal renal function. Long-term treatment with oral fluconazole and triple immunosuppression therapy in which the conventional dose of tacrolimus was reduced by half was indicated. After 12 months posttransplant, fluconazole was reduced, and the dose of tacrolimus was adjusted in consequence. After 18 months post-transplant, the patient continued with normal renal function and no associated symptoms. He has been followed routinely as an outpatient, and as of July 2018 continues with normal renal function. He has gone back to his usual activities and is still very keen to go hunting again although his doctors are not.

\section{Discussion}

Clinical manifestations of opportunistic diseases in transplant patients are frequently non-specific and mostly not early recognized. In the reported case, the diagnosis of pulmonary coccidioidomycosis was a result of an intense evaluation protocol in which more common causes of infection in the transplant patient were ruled-out. The early histological diagnosis, facilitated by the video-assisted lung biopsy, allowed the medical team to establish a specific treatment for a systemic mycosis, traditionally associated with a high morbidity and mortality rate in transplanted patients. The incidence of coccidioidomycosis has risen substantially during the past two decades. Although the incidence of this infection decreased slightly during 2007-2008, it then increased dramatically from $154 / 100,000$ during 2009 to $248 / 100,000$ during 2011 [9]. Reasons for the observed increase since 2009 are not known but may be due in part to that major commercial laboratory changed their reporting practices to conform with the other laboratories in Arizona and are now reporting positive enzyme immunoassay (EIA) results as cases without additional confirmation by immunodiffusion [10]. However, the possibility of a real and significant increase in the natural occurrence of this infection is still a hypothesis to test.

Coccidiodomycosis has been described after lung, kidney, heart and liver transplantation with an incidence of $1.4-6.9 \%$ in endemic regions $[11,12]$. As it is accepted that no person-to-person transmission of the pathogen occurs, except in cases of in utero exposure [13] or in a recipient-acquired infection from an organ donor during solidorgan transplantation [2,6], the occurrence of coocdidiodomycosis in transplant recipients living outside of endemic regions is produced after arthroconidia are aerolized from soil and inhaled into the lungs $[1,12]$. Among this population, the severity of Coccidioides infection correlates with the underlying immune status [12]. Most of these infections are diagnosed within the first year of transplant and they are believed to most commonly result from reactivation of previously acquired coccidioidomycosis [11]. Risk factors for post-transplant coccidioidomycosis include a previous history of coccidioidomycosis, positive serology at the time of transplantation, and the use of antirejection therapy [11,14-17]. In the case reported here, although there was no solid evidence of a previous clinical episode of coccidiodomycosis, it is highly possible that the occurrence of this episode of pulmonary infection was the result from a previous lung exposure to arthroconidia during a former hunting expedition to endemic zones in the North of Mexico. Unfortunately, neither intradermoreaction for Coccidioides (coccidiodina test) nor serology for 

condition

Coccidioides ssp are routinely performed as part of the pre-transplant evaluation protocol in any transplant center of Mexico City, a nonendemic region for Coccidiodomycosis. Whether any of these tests would be accurately ruled-out/confirmed the presence of a quiescent chronic Coccidiodes infection and the need for pre-transplant antimycotic treatment is as question that had risen an interesting debate that is, however, outside of the reach of this report. Solid organ transplant recipients require lifelong or very heavy suppression of cell-mediated immunity to prevent or treat organ rejection. Both conditions increase the risk of coccidioidal infection or other opportunistic infections in transplant patients [11,18,19]. In the case reported here, immunosuppressive induction was based in a nondepleting monoclonal antibody (basiliximab), maintenance therapy was based on traditional immunosuppressive drugs and doses and no evidence of acute rejection was clinically suspected nor documented.

In general, $60 \%$ of patients infected with Coccidioides ssp are asymptomatic and can only be detected by the expression of a cellular immune reaction to a coccidioidal antigen. The other $40 \%$ will present with primary pulmonary coccidioidomycosis and it is estimated that perhaps $1-5 \%$ will develop extrathoracic dissemination. Primary pulmonary disease is a usually sub-acute and self-limited syndrome, characterized by fatigue, cough, chest pain, dyspnea and, occasionally, hemoptysis [20]. The manifestations appear between 10 and 15 days after exposure to the fungus, and the intensity of the symptoms depends directly on the infective load, ranging from the flu to a severe, nonspecific respiratory infection, accompanied by high fever, chest pain and cough with or without expectoration, as well as by general symptoms or allergic manifestations, particularly erythema nodosum [20]. Primary pulmonary coccidioidomycosis generally resolves spontaneously within 30-60 days, even without antifungal treatment, and patients retain lifelong immunity to exogenous infection [20,21]. In contrast, people with compromised immune systems such as patients with lymphoma, organ transplants or AIDS are more likely to develop severe pneumonia and disseminated infection $[11,12,22,23]$ and particularly susceptible to chronic forms of pulmonary disease $[24,25]$. This disease is characterized by pulmonary cavitation but the lungs might also be diffusely affected because of the inhalation of a large quantity of infective arthroconidia or as a late and secondary presentation resulting from hematogenous dissemination. These disseminated forms present with multiple diffuse infiltrates, the largest of which might present cavitation, which can lead to severe respiratory manifestations and respiratory failure.

The disease might progress to death, mimicking septic shock, accompanied by acute respiratory distress syndrome, with high mortality rates $[24,26]$.

Radiographic findings are varied and may consist of one or more of the following: lobar consolidation, pulmonary nodules, mass-like lesions, interstitial infiltrates or cavitary disease $[11,15]$.

In endemic areas, recipients usually present with common and non-specific symptoms as fever, chills, night sweats, cough, dyspnea and pleurisy within 1 month following transplantation; hence, in these cases coccidioidomycosis is always considered in the differential diagnosis [27]. In contrast, in non-endemic areas, the latter symptoms are traditionally attributed to more common agents, and there is highly probable that coccidiodomycosis is not considered in the initial deferential diagnosis, as occurred in the presented case.

Treatment choice, dose, and duration of antifungal therapy depend on the severity of illness, and the extent and location of the infection $[28,29]$. Acute pulmonary coccidioidomycosis may be mild and self- limited in the immunocompetent host and antifungal therapy may be withheld with close clinical monitoring [12]. However, all patients with underlying immune impairment, including organ transplant recipients, must be treated regardless of the severity of infection [27]. Treatment options for most patients with mild to moderate coccidioidomycosis include oral fluconazole or itraconazole [12,30]. Where infections involve the central nervous system, higher doses of fluconazole (such as $800 \mathrm{mg}$ daily) may be required, since it has excellent CSF penetration [27,28]. Musculoskeletal infections may be best treated with itraconazole [12]. Fluconazole and other azoles inhibit cytochrome P-450 enzymes, leading to increased concentration of calcineurin inhibitors (such as cyclosporine or tacrolimus) and enhanced risk of nephrotoxicity or other adverse effects. Therefore, close therapeutic drug level monitoring of the calcineurin inhibitors is recommended after initiating or altering the dose of azole antifungals [28]. As occur in the presented case, for rapidly progressing or lifethreatening coccidioidomycosis, amphotericin B is selected as initial therapy because its antifungal effect appears to have a more rapid onset than that of oral azoles $[11,28]$. During early experience with the management of coccidioidomycosis in transplant recipients, patients were noted to develop relapsing or recurrent disease after initial control of their infection [11]. Lifelong antifungals were subsequently used successfully as secondary prophylaxis to prevent such relapses [31]. Organ transplant recipients must be treated regardless of the severity of infection [12] and lifelong antifungal prophylaxis is recommended once active coccidioidomycosis has been controlled to prevent further relapses [28,31].

\section{Conclusion}

Coccicidyodal infection is a rare disease outside endemic areas which can prove potentially fatal if not suspected. People who live in or travel to an endemic region can become infected and the time-frame for clinical appearance and severity correlates with the underlying immune status, being possible the apparition of symptoms months to years after the exposure to the fungi. An intense diagnostic approach should be performed after ruling out more common causes of infection in posttransplant patients in order to detect the infection. Treatment should be started immediately after diagnosis to prevent the morbidity and mortality associated with the disease. Nonetheless, the lack of suspicion of the disease and the difficulty of the diagnosis in poor resource settings makes the infection challenging for the immunocompromised patient and its medical team and prompts for further investigation in diagnostic tools and methods of detection in this particular context in order to treat a potentially lethal condition.

\section{References}

1. Brown J, Benedict K, Park BJ, Thompson GR (2013) Coccidioidomycosis: epidemiology. Clin Epidemiol 5: 185-197. [Crossref]

2. Cole GT, Hurtgen BJ, Hung CY (2012) Progress Toward a Human Vaccine Against Coccidioidomycosis. Curr Fungal Infect Rep 6: 235-244.

3. Thompson GR 3rd (2011) Pulmonary coccidioidomycosis. Semin Respir Crit Care Med 32: 754-763.

4. Galgiani JN, Ampel NM, Blair JE, Catanzaro A, Johnson RH, et al. (2005) Coccidioidomycosis. Clin Infect Dis 41: 1217-1223. [crossref]

5. Ampel NM (2010) New perspectives on coccidioidomycosis. Proc Am Thorac Soc 7 181-185. [Crossref]

6. Gobbi F, Angheben A, Farina C, Buonfrate D, Postiglione C, et al. (2012) Coccidioidomycosis: first imported case in Italy. J Travel Med 19: 192-194. [Crossref]

7. Sondermeyer G, Lee L, Gilliss D, Tabnak F, Vugia D (2013) Coccidioidomycosisassociated hospitalizations, California, USA, 2000-2011. Emerg Infect Dis 19:1590-1597. 

condition

8. Chiller TM, Galgiani JN, Stevens DA (2003) Coccidioidomycosis. Infect Dis Clin North Am 17: 41-57. [Crossref]

9. Centers for Disease Control and Prevention (CDC) (2013) Increase in reported coccidioidomycosis - United States, 1998-2011. MMWR Morb Mortal Wkly Rep 62: 217-221.

10. Hector RF, Rutherford GW, Tsang CA, Erhart LM, McCotter O, et al. (2011) The public health impact of coccidioidomycosis in Arizona and California. Int $J$ Environ Res Public Health 8: 1150-1173. [Crossref]

11. Blair JE, Logan JL (2001) Coccidioidomycosis in solid organ transplantation. Clin Infect Dis 33: 1536-1544. [Crossref]

12. Miller R, Assi M (2013) AST Infectious Diseases Community of Practice. Endemic fungal infections in solid organ transplantation. Am J Transplant 4: 250-261.

13. Bercovitch, Robert S (2011) "Coccidioidomycosis during pregnancy: a review and recommendations for management." Clin Infect Dis 53.4: 363-368.

14. Blair JE (2007) Coccidioidomycosis in patients who have undergone transplantation. Ann N Y Acad Sci 1111: 365-376. [Crossref]

15. Blair JE (2006) Coccidioidomycosis in liver transplantation. Liver Transpl 12: 31-39. [Crossref]

16. Braddy CM, Heilman RL, Blair JE (2006) Coccidioidomycosis after renal transplantation in an endemic area. Am J Transplant 6: 340-345. [Crossref]

17. Vikram HR, Dosanjh A, Blair JE (2011) Coccidioidomycosis and lung transplantation. Transplantation 92: 717-721. [Crossref]

18. Vucicevic D, Carey EJ, Blair JE (2011) Coccidioidomycosis in liver transplant recipients in an endemic area. Am J Transplant 11: 111-119. [Crossref]

19. Mendoza N, Blair JE (2013) The utility of diagnostic testing for active coccidioidomycosis in solid organ transplant recipients. Am J Transplant 13: 1034-1039.
20. Ampel NM, Mosley DG, England B, Vertz PD, Komatsu K, et al. (1998) Coccidioidomycosis in Arizona: increase in incidence from 1990 to 1995. Clin Infect Dis 27: 1528-1530. [Crossref]

21. Veras KN, Figueirêdo BC, Martins LM, Vasconcelos JT, Wanke B (2003) Coccidioidomycosis: a rare cause of respiratory distress syndrome. J Pneumol 29: 45-48.

22. Braddy CM, Heilman RL, Blair JE (2006) Coccidioidomycosis after renal transplantation in an endemic area. Am J Transplant 6: 340-345. [Crossref]

23. Tepeoglu M, Erinanc H, Ozdemir H, Turan H, Moray G, et al. (2013) Pulmonary Coccidioidomycosis After a Renal Transplant in a Nonendemic Region. Exp Clin Transplant 12: 71-73.

24. Galgiani JN (1999) Coccidioidomycosis: a regional disease of national importance. Rethinking approaches for control. Ann Intern Med 130: 293-300.

25. Stevens DA (1995) Coccidioidomycosis. N Engl J Med 332: 1077-1082. [Crossref]

26. Mayorga RP, Espinoza H (1970) Coccidioidomycosis in Mexico and Central America. Mycopathol Mycol Appl 41: 13-23. [Crossref]

27. Galgiani J, Catanzaro A, Cloud GA (2000) Comparison of oral fluconazole and itraconazole for progressive, nonmeningeal coccidioidomycosis. A randomized, double-blind trial. Mycoses Study Group. Ann Intern Med 133: 676-686.

28. Keckich D, Blair JE, Vikram HR, Seville MT, Kusne S (2011) Reactivation of coccidioidomycosis despite antifungal prophylaxis in solid organ transplant recipients. Transplantation 92: 88-93.

29. Galgiani JN, Ampel NM, Catanzaro A, Johnson RH, Stevens DA, et al. (2000) Practice guideline for the treatment of coccidioidomycosis. Infectious Diseases Society of America. Clin Infect Dis 30: 658-661.

30. Vikram HR, Blair JE (2009) Coccidioidomycosis in transplant recipients: a primer for clinicians in nonendemic areas. Curr Opin Organ Transplant 14: 606-612. [Crossref]

31. Galgiani JN, Ampel NM, Blair JE, Catanzaro A, Johnson RH, et al. (2005) Coccidioidomycosis. Clin Infect Dis 41: 1217-1223. [Crossref]

Copyright: (C2018 Leyva-Córdova D. This is an open-access article distributed under the terms of the Creative Commons Attribution License, which permits unrestricted use, distribution, and reproduction in any medium, provided the original author and source are credited. 